\title{
Effects of not intubating non-trauma patients with low Glasgow Coma Scale scores: A retrospective study
}

Loui Alsulimani, Ohoud Baajlan, Khalid Alghamdi, Raghad Alahmadi, Abdullah Bakhsh, and Jameel Abualenain

Abstract-Background: Endotracheal intubation (EI) is a critical life-saving procedure commonly performed on emergency department (ED) patients who present with altered mental status (AMS).

Aims: We aimed to investigate the safety of observing, without EI, patients who present to the ED with decreased levels of consciousness (LOC).

Methods: We reviewed the data of all adult ED patients with a Glasgow Coma Scale (GCS) score $\leq 8$, during the period between 2012 and 2018, in an academic tertiary care centre. Trauma patients were excluded. The patients were divided into two groups for comparison: those who were intubated and those who were not. Data on mortality, morbidity, and baseline clinical characteristics were collected and analysed.

Results: After screening 6334 electronic medical records of patients presenting to the ED with decreased LOC, only 257 patients met the inclusion criteria. $173(67.3 \%)$ patients were intubated, while 84 $(32.7 \%)$ were not. Among the intubated patients, 165 (95.4\%) were intubated early (within two hours of presentation). Mortality, morbidity and length of stay for the intubated group were higher, although the baseline clinical characteristics were the same.

Conclusion: It might be safe to observe non-trauma emergency patients with a GCS score $\leq 8$ without intubation. However, such decision should be taken carefully, as delayed intubation can be associated with higher mortality and morbidity.

Loui Alsulimani, Ohoud Baajlan, Abdullah Bakhsh, Jameel Abualenain are with Department of Emergency Medicine, Faculty of Medicine, King Abdulaziz University, e-mail: lkalsulimani@kau.edu.sa, e-mail: ohoudbaajlan@gmail.com, e-mail: aarbakhsh@kau.edu.sa, e-mail: jameel.abualenain@gmail.com

Loui Alsulimani is the corresponding author.

Khalid Alghamdi (e-mail: dr.kh.ghamdi@gmail.com)

Raghad Alahmadi (e-mail: raghadsaleh13@gmail.com)
Index Terms - Endotracheal intubation, Intubation in the ED, Non-trauma patients with low Glasgow Coma Scale scores

\section{INTRODUCTION}

Endotracheal intubation (EI) in acute care settings is generally a stress-provoking and high-stakes procedure, often with no clear cut-off point regarding its indication. Although it has an excellent success rate ( 100\%) [1], serious complications such as oxygen desaturation, cardiac arrest, oesophageal intubation, and vomiting/regurgitation [2] can occur, even under expert care. Most intubated patients are admitted to the intensive care unit (ICU), which is costly (around 4300 US Dollars per day) [3]. Therefore, EI must be performed for the correct indications; namely, apnoea, respiratory failure, airway obstruction, loss of airway reflexes, decreased levels of consciousness (LOC), haemodynamic instability, and cardiopulmonary arrest [4]. Most EIs are for medical cases $[5,6]$, including severe cognitive impairment, hypoventilation, and airway obstruction $[7,8]$.

This study aimed to investigate the effects of observing non-trauma patients with a Glasgow Coma Scale (GCS) score $\leq 8$ without intubating them during their ED visit, and compare these with patients who were intubated, in terms of complications and mortality. Early EI was defined as intubation performed during the first two hours after arrival at the ED $[8,9]$; a specific focus was given to identify this group.

\section{Materials And Methods}

We conducted a retrospective chart review to investigate the safety of observing unconscious nontrauma patients with decreased LOC who presented 
to the ED in an academic tertiary care university centre. We reviewed the electronic medical records of all adult patients ( $\geq 14$ years according to the hospital system) who presented to the ED between January 2012 and December 2018 with decreased LOC.

The LOC of patients presenting to the hospital's ED is usually recorded electronically in the triage notes, using the AVPU scale, by the triage nurses during the initial encounter. The patient's primary nurse then records the GCS once the patient is assigned to a treatment area. For this study, data from the Health Information System (HIS) were retrieved by the hospital's statistics unit and the HIS data of all patients with decreased LOC whose AVPU scale was recorded as verbal (V), unresponsive (U), or pain (P) [i.e., all categories excluding alert (A)] were separated. Subsequently, the patients' electronic medical records (EMRs) were reviewed by the research team to obtain the GCS scores from the nurses' notes. The research team created an electronic data collection sheet to record the study variables and established and trained a data collection team for data retrieval. The research team supervised the data collection team and reviewed the collected data for any inconsistencies or noncompliance with requirements.

All patients with a GCS score $\leq 8$ were included in the study; those who presented with trauma, cardiac arrest, and do-not-resuscitate status were excluded. The eligible study population (those with a GCS score $\leq 8$ ) were divided into two groups: those who were intubated and those who were not intubated during the studied period of hospitalization. The main goal was to compare mortality and morbidity between the two groups. For this purpose, data were collected manually by reviewing the saved documents. Study data were collected into an electronic data collection sheet, from a secure database only accessible to the research team. After the data collection, any patient identifiers were deidentified for confidentiality and the original data were kept by the primary investigator in an encrypted device. For statistical analysis, IBM SPSS Statistics for Windows, version 21.0 (IBM Corp., Armonk, N.Y., USA) was used. Continuous variables were expressed as mean (standard deviation [SD]), whereas categorical variables were expressed as numbers (percentages). Differences among the continuous variables were compared using Student's t-tests, and those among categorical variables were compared using either the chi-square or Fisher's exact test. In statistical testing, a two-sided $\mathrm{P}<0.05$ was considered statistically significant.

\section{RESULTS}

Of the 6334 patients who presented to the ED with decreased LOC between January 2012 and December 2018, 4056 were adults ( $\geq 14$ years). Only 257 patients met the inclusion criteria with a GCS score $\leq 8$ and were included in the final analysis (Figure 1). Of these, $173(67.3 \%)$ were intubated, while the remaining 84 (32.7\%) were not. Of the 173 patients in the intubated group, most (165 [95.4\%]) were intubated early, i.e., within two hours of presentation to the ED, whereas eight $(4.6 \%)$ were intubated more than two hours after presentation (two on the first day and six on the second day).

The total mortality and all-cause morbidity among the 257 patients included in the study were $28.2 \%$ and $22.6 \%$ respectively. $149(58 \%)$ patients were male, and $191(74.3 \%)$ had at least one pre-existing comorbidity. The mean length of hospital stay for all 257 patients was 12.5 days. The mean age of included patients was 63.9 years (SD +/- 18.81). The mean GCS score at the time of presentation was 5.51 (median 6), and the most frequent (mode) was 3. Furthermore, there was a correlation between the AVPU responsiveness scale and the GCS score. Patients who presented as unresponsive (U) had a lower average GCS score (5) than those who were responsive to pain (P) (average GCS score of 6) or verbal (V) (average GCS score of 7) stimuli.

A. Demographics: The clinical characteristics of the two groups (intubated and non-intubated) are illustrated in Table 1. In both groups, most of the patients who presented with low GCS scores were around 60 years old. Moreover, more than half of the patients in both groups were male. It was observed that the lower the GCS score from 8, the more likely it was that the patient would be intubated, and vice versa $(\mathrm{P}$-value $<0.05)$. Intubation was more common in patients presenting with neurological disorders than in those presenting with other pathologies, cerebrovascular accident being 


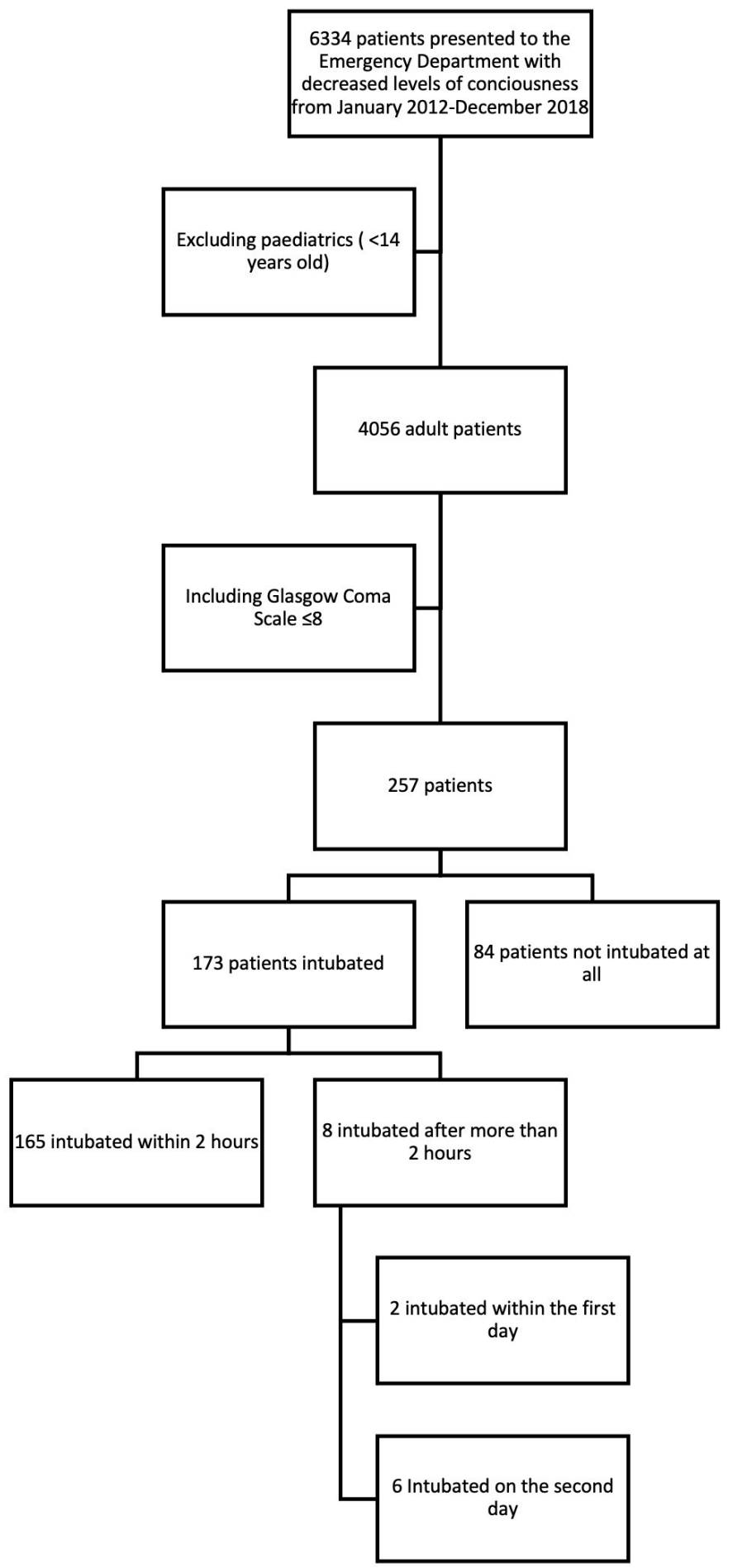

Fig. 1. Flow chart of research steps 
the most common reason. Hypertension was the most frequent comorbidity in both groups, followed by diabetes mellitus and a previous history of stroke (Figure 2). Other comorbidities were also observed which, according to this study's protocol, were not considered major comorbidities, such as liver cirrhosis, benign prostate hypertrophy and Parkinson's disease, among others.

B. Patients' outcomes: There was no statistically significant difference in mortality or morbidity between the two groups: Mortality was $28.7 \%$ in the intubated group vs. $27.2 \%$ in the non-intubated group, and all-cause morbidity (aspiration pneumonia, hypoxic brain injury, cerebral oedema and major organ failure) $24.9 \%$ in the intubated group vs. $17.9 \%$ in the non-intubated group (Table 2). All organ failure during hospital admission was higher in the intubated group than in the non-intubated group; however, also not statistically significant (Figure 3). Most patients who were intubated required a prolonged (more than 14 days) admission to the ICU, whereas only a quarter of patients who were not intubated needed ICU admission within 14 days of presentation to the ED. The length of stay was longer for the intubated group (almost 13 days); the nonintubated group spent approximately two days less than the intubated group.

\section{Discussion}

In this study, we demonstrated the possibility of safely observing medical emergency patients who present to the ED with a GCS score $\leq 8$, without performing intubation. Almost one-third (32.7\%) of these patients were not intubated throughout their hospital visit, and this group had lower morbidity rates than those who were intubated. However, mortality rates were similar in both groups. On analyzing certain baseline characteristics (including age, GCS score upon presentation, and comorbidities) of both the intubated and observation groups, no significant variability was found; this minimises possible bias due to baseline variability between the compared groups.
A review of the literature on this topic revealed previous studies showing similar results for intoxicated patients with comparable presentation $[7,10$, 11]. Duncan et al showed no evidence of complications when patients with alcohol intoxication and a GCS score $\leq 8$ were observed until sobriety was regained [11]. This indicates that patients with temporary or reversible conditions causing a GCS score $\leq 8$ may not necessarily require early intubation. This study demonstrates that the majority of patients $(91.3 \%)$ who were deemed by emergency physicians not to require early intubation most likely did not require it. Regarding the eight $(8.7 \%)$ patients who did require subsequent intubation, it is unlikely that the cause was physicians' misjudgment, since most of these patients (six out of eight) were able to tolerate non-intubation for at least 24 hours. Overall, the patients requiring subsequent intubation only represent a small percentage of those who were not intubated within the first two hours. The AVPU (Alert, responds to Voice, responds to Pain, Unresponsive) scale and the Glasgow Coma Scale (GCS) are the common tools used to assess LOC [10, $12,13]$. The AVPU scale is a simple and easy tool to rapidly assess altered mental status (AMS). However, it is insufficient for early detection of changes in LOC, less comprehensive for assessing all the levels of an AMS, and difficult to obtain in some intoxicated patients [13]. The GCS, meanwhile, was initially used for traumatic head injuries but has now become a common tool for the subjective measurement of LOC $[13,14]$. GCS scores and gag reflexes are poorly related; hence the gag reflex may be normal or diminished at any GCS score $[15,16]$. The presence of a gag reflex is a very specific criterion for the exclusion of intubation, but not vice versa [17]. Nonetheless, the airway should be assessed separately from LOC $[15,16]$.

Although a GCS score $\leq 8$ is a well-known indicator for intubation in trauma patients, it is still controversial in alcohol-intoxicated, drug-poisoned, and non-trauma patients [7]. A study of poisoned patients found that the rate of complications in those with low GCS scores was low; therefore, the "GCS 8 , intubate" rule likely does not apply to these patients [11]. Some studies assert that using a GCS score $\leq 8$ as an indication for intubation in intoxicated patients is not appropriate, as those patients 
Table 1. Demographics and clinical characteristics of patients

\begin{tabular}{|c|c|c|c|c|c|c|c|}
\hline \multirow[t]{3}{*}{ Demographic } & & \multicolumn{6}{|c|}{ Intubation status } \\
\hline & & \multicolumn{2}{|c|}{ Intubated } & \multicolumn{2}{|c|}{ Not intubated } & \multirow[t]{2}{*}{ Missing } & \multirow[t]{2}{*}{ P-value } \\
\hline & & Count & $\begin{array}{l}\text { Column } \\
\mathrm{N} \%\end{array}$ & Count & $\begin{array}{l}\text { Column } \\
\mathrm{N} \%\end{array}$ & & \\
\hline \multirow[t]{2}{*}{ Age } & Count & 171 & ---- & 84 & ---- & 2 & 0.069 \\
\hline & Mean & \multicolumn{2}{|c|}{$\begin{array}{l}62.47 \text { years } \\
(\mathrm{SD} 17.78)\end{array}$} & \multicolumn{2}{|c|}{$\begin{array}{l}67.04 \text { years }(\mathrm{SD} \\
20.53)\end{array}$} & & \\
\hline \multirow{4}{*}{$\begin{array}{l}\text { GCS on } \\
\text { presentation }\end{array}$} & Count & 173 & ---- & 84 & ---- & 0 & 0.049 \\
\hline & Mean & $5.36(\mathrm{~S}$ & 1.69) & $5.82(\mathrm{~S}$ & 1.89) & & \\
\hline & Median & 6 & & 6 & & & \\
\hline & Mode & 6 & & 7 & & & \\
\hline \multirow[t]{2}{*}{ Gender } & Male & 109 & $63.0 \%$ & 40 & $47.6 \%$ & 0 & 0.019 \\
\hline & Female & 64 & $37.0 \%$ & 44 & $52.4 \%$ & & \\
\hline \multirow{5}{*}{$\begin{array}{l}\text { Final } \\
\text { Diagnosis }\end{array}$} & Neurological & 73 & $45.6 \%$ & 27 & $35.1 \%$ & 20 & 0.319 \\
\hline & $\begin{array}{l}\text { Abdominal } \\
\text { and renal }\end{array}$ & 21 & $13.1 \%$ & 10 & $13.0 \%$ & & \\
\hline & Infectious & 36 & $22.5 \%$ & 27 & $35.1 \%$ & & \\
\hline & Respiratory & 23 & $14.4 \%$ & 11 & $14.3 \%$ & & \\
\hline & Cardiac & 7 & $4.4 \%$ & 2 & $2.6 \%$ & & \\
\hline
\end{tabular}

GCS: Glasgow Coma Scale score

Table 2. Patients' hospital outcomes

\begin{tabular}{|c|c|c|c|c|c|c|c|}
\hline \multicolumn{2}{|c|}{ Hospital course } & \multicolumn{6}{|c|}{ Intubation status } \\
\hline & & \multicolumn{2}{|c|}{ Intubated } & \multicolumn{2}{|c|}{ Not intubated } & \multirow[t]{2}{*}{ Missing } & \multirow[t]{2}{*}{ P-value } \\
\hline & & Count & $\begin{array}{l}\text { Column } \\
\mathrm{N} \%\end{array}$ & Count & $\begin{array}{l}\text { Column } \\
\mathrm{N} \%\end{array}$ & & \\
\hline \multirow[t]{2}{*}{ Mortality } & Yes & 47 & $28.7 \%$ & 22 & $27.2 \%$ & \multirow[t]{2}{*}{12} & \multirow[t]{2}{*}{0.806} \\
\hline & No & 117 & $71.3 \%$ & 59 & $72.8 \%$ & & \\
\hline \multirow[t]{2}{*}{ Morbidity } & Yes & 43 & $24.9 \%$ & 15 & $17.9 \%$ & \multirow[t]{2}{*}{0} & \multirow[t]{2}{*}{0.208} \\
\hline & No & 130 & $75.1 \%$ & 69 & $82.1 \%$ & & \\
\hline \multirow{2}{*}{$\begin{array}{l}\text { All organ } \\
\text { failure }\end{array}$} & Yes & 18 & $10.4 \%$ & 4 & $4.8 \%$ & \multirow[t]{2}{*}{0} & \multirow[t]{2}{*}{0.158} \\
\hline & No & 155 & $89.6 \%$ & 80 & $95.2 \%$ & & \\
\hline
\end{tabular}




\begin{tabular}{|c|c|c|c|c|c|c|c|c|}
\hline \multirow{4}{*}{ Dispatch } & \multicolumn{2}{|l|}{ ICU } & 98 & $62.8 \%$ & 18 & $25.4 \%$ & 30 & 0.000 \\
\hline & \multicolumn{2}{|c|}{ Medical } & 39 & $25.0 \%$ & 26 & $36.6 \%$ & & \\
\hline & \multicolumn{2}{|c|}{ Surgical } & 8 & $5.1 \%$ & 4 & $5.6 \%$ & & \\
\hline & \multicolumn{2}{|c|}{ Discharged } & 11 & $7.1 \%$ & 23 & $32.4 \%$ & & \\
\hline \multirow{2}{*}{$\begin{array}{l}\text { Length of } \\
\text { stay }\end{array}$} & Mean & SD & Mean & SD & Mean & SD & 31 & 0.553 \\
\hline & $\begin{array}{l}12.46 \\
\text { days }\end{array}$ & \pm 24.68 & 13.14 & \pm 21.19 & 11.05 & \pm 30.75 & & \\
\hline
\end{tabular}

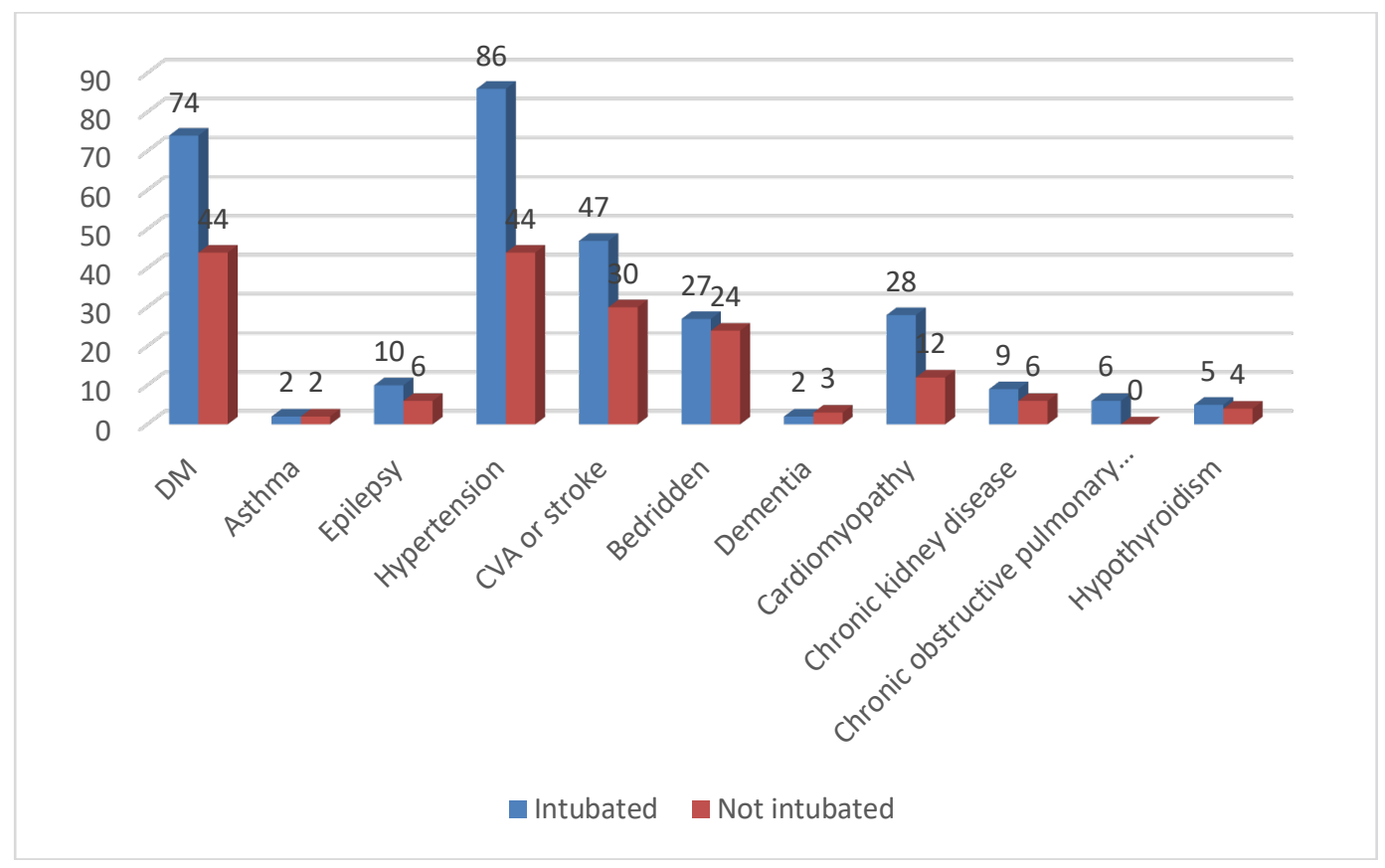

Fig. 2. Patients' comorbidities on presentation

may be able to maintain ventilation and protect their airway unassisted $[7,10,11]$. In contrast, Chan et al concluded that a GCS score $\leq 8$ is a sensitive indicator for intubation in poisoned patients [17].

Various scores have been used to aid the decision to intubate patients with decreased LOC. For example, the Bispectral Index (BIS) was described as a useful tool to determine the need for intubation in opioid-poisoned patients [18]. In this study, we used mainly the GCS and AVPU scores. The AVPU values correspond to GCS scores of $15(\mathrm{~A}), 13(\mathrm{~V}), 8(\mathrm{P})$, and $3(\mathrm{U})$, respectively, with narrow ranges and some overlap [13]. Interestingly, this study showed a similar pattern of correlation between the two scores. Moreover, the closer to 8 the GCSscore, the more likely it could be for the physician to avoid early intubation if the clinical condition persists. It is important to note that the mean and median GCS scores in both groups were similar. This equality in the baseline GCS scores of both groups gives more validity to the findings of this study by minimizing variation in a major baseline characteristic.

This study has some limitations. The relatively small sample size overall, and especially in the delayed intubation group, may limit the generalizability of the results. Future studies should include a larger sample size and use of specific diagnoses for more clinical applicability. Furthermore, this 
study does not include data relating to vital signs and blood gas results, as these were available in paperbased documents, making them difficult to retrieve and creating the problem of a large proportion of missed data. It is not clear whether these variables would have an effect on the study's outcome. Moreover, it was difficult to determine from the EMRs whether the low GCS score at presentation was a baseline score for the patient or an acute presentation. Nevertheless, we believe that the study addresses the concept of safety of observation (without intubation) in general. Further studies are needed to address the abovementioned limitations.

\section{Conclusion}

Although most non-trauma patients presenting to the ED with a GCS score $\leq 8$ require intubation within the critical initial few hours after presentation, a significant percentage can be safely observed without undergoing intubation. If patients with a GCS score $\leq 8$ can safely pass the critical first two hours after presentation without requiring intubation, it is unlikely that they will need intubation later during their hospitalization. However, emergency physicians should be careful in selecting the right candidates for observation without intubation if the GCS score is $\leq 8$.

In conclusion, the physician's clinical assessment plays a paramount role in deciding whether patients with a GCS score $\leq 8$ need immediate intubation or further observation without intubation. Many clinical parameters may aid this decision. Clinical decision protocols are required that follow the best available guidance, based on existing knowledge, clinical judgment, and hospital policies, to assist ED physicians during such critical and stressful moments. Further exploration of variables from this study and others may help guide the development of such beneficial tools.

\section{SOURCE OF FUNDING:}

No funding was received.

\section{CONFLICT OF INTEREST:}

The authors have no conflicts of interest.

\section{ETHICAL APPROVAL}

This research was approved by the Unit of Biomedical Research Ethics Committee in the Faculty of Medicine (Reference No. 584-18) in November 2018 .

\section{ACKnOWledgments}

We would like to thank the data collection team. We also thank Editage by Cactus ${ }^{\circledR}$ for editing the manuscript.

\section{REFERENCES}

1. Bernhard M, Bax SN, Hartwig T, YahiaouiDoktor M, Petros S, Bercker S, et al. Airway Management in the Emergency Department (The OcEAN-Study) - a prospective single centre observational cohort study. Scandinavian journal of trauma, resuscitation and emergency medicine. 2019;27(1):1-9.

2. Ono Y, Kakamu T, Kikuchi H, Mori Y, Watanabe Y, Shinohara K. Expert-performed endotracheal intubation-related complications in trauma patients: incidence, possible risk factors, and outcomes in the prehospital setting and emergency department. Emergency medicine international. 2018;2018.

3. Halpern NA, Pastores SM. Critical care medicine beds, use, occupancy and costs in the United States: a methodological review. Critical care medicine. 2015;43(11):2452.

4. Şahiner Y. Indications for Endotracheal Intubation. Tracheal Intubation 2018.

5. Brown III CA, Bair AE, Pallin DJ, Walls RM, Investigators NI. Techniques, success, and adverse events of emergency department adult intubations. Annals of Emergency Medicine. 2015;65(4):363-70. e1.

6. Alkhouri H, Vassiliadis J, Murray M, Mackenzie J, Tzannes A, McCarthy S, et al. Emergency airway management in Australian and New Zealand emergency departments: a multicentre descriptive study of 3710 emergency intubations. Emergency Medicine Australasia. 2017;29(5):499-508.

7. Sauter TC, Rönz K, Hirschi T, Lehmann B, Hütt $\mathrm{C}$, Exadaktylos AK, et al. Intubation in acute alcohol intoxications at the emergency department. Scandinavian journal of trauma, resuscitation and emergency medicine. 2020;28(1):1-10.

8. Sise MJ, Shackford SR, Sise CB, Sack DI, Paci GM, Yale RS, et al. Early intubation in the management of trauma patients: indications and outcomes in 1,000 consecutive patients. Journal of 
Trauma and Acute Care Surgery. 2009;66(1):32-40.

9. Trupka A, Waydhas C, Nast-Kolb D, Schweiberer L. Early intubation in severely injured patients. European journal of emergency medicine: official journal of the European Society for Emergency Medicine. 1994;1(1):1-8.

10. Rajabi Kheirabadi A, Tabeshpour J, Afshari R. Comparison of three consciousness assessment scales in poisoned patients and recommendation of a new scale: AVPU plus. Asia Pacific Journal of Medical Toxicology. 2015;4(2):58-63.

11. Duncan R, Thakore S. Decreased Glasgow Coma Scale score does not mandate endotracheal intubation in the emergency department. The journal of emergency medicine. 2009;37(4):451-5.

12. Romanelli D, Farrell MW. AVPU (alert, voice, pain, unresponsive). Treasure Island: StatPearls Publishing. 2019.

13. Kelly CA, Upex A, Bateman DN. Comparison of consciousness level assessment in the poisoned patient using the alert/verbal/painful/unresponsive scale and the Glasgow Coma Scale. Annals of emergency medicine. 2004;44(2):108-13.
14. Zadravecz FJ, Tien L, Robertson-Dick BJ, Yuen TC, Twu NM, Churpek MM, et al. Comparison of mental-status scales for predicting mortality on the general wards. Journal of hospital medicine. 2015;10(10):658-63.

15. Moulton C, Pennycook A, Makower R. Relation between Glasgow coma scale and the gag reflex. BMJ: British Medical Journal. 1991;303(6812):1240.

16. Moulton C, Pennycook AG. Relation between Glasgow coma score and cough reflex. The Lancet. 1994;343(8908):1261-2.

17. Chan B, Gaudry P, Grattan-Smith T, McNeil R. The use of Glasgow Coma Scale in poisoning. The Journal of emergency medicine. 1993;11(5):579-82.

18. Eizadi-Mood N, Yaraghi A, Alikhasi M, Jabalameli M, Farsaei S, Sabzghabaee AM. Prediction of endotracheal intubation outcome in opioid-poisoned patients: A clinical approach to bispectral monitoring. Canadian journal of respiratory therapy: CJRT $=$ Revue canadienne de la therapie respiratoire: RCTR. 2014;50(3):83.

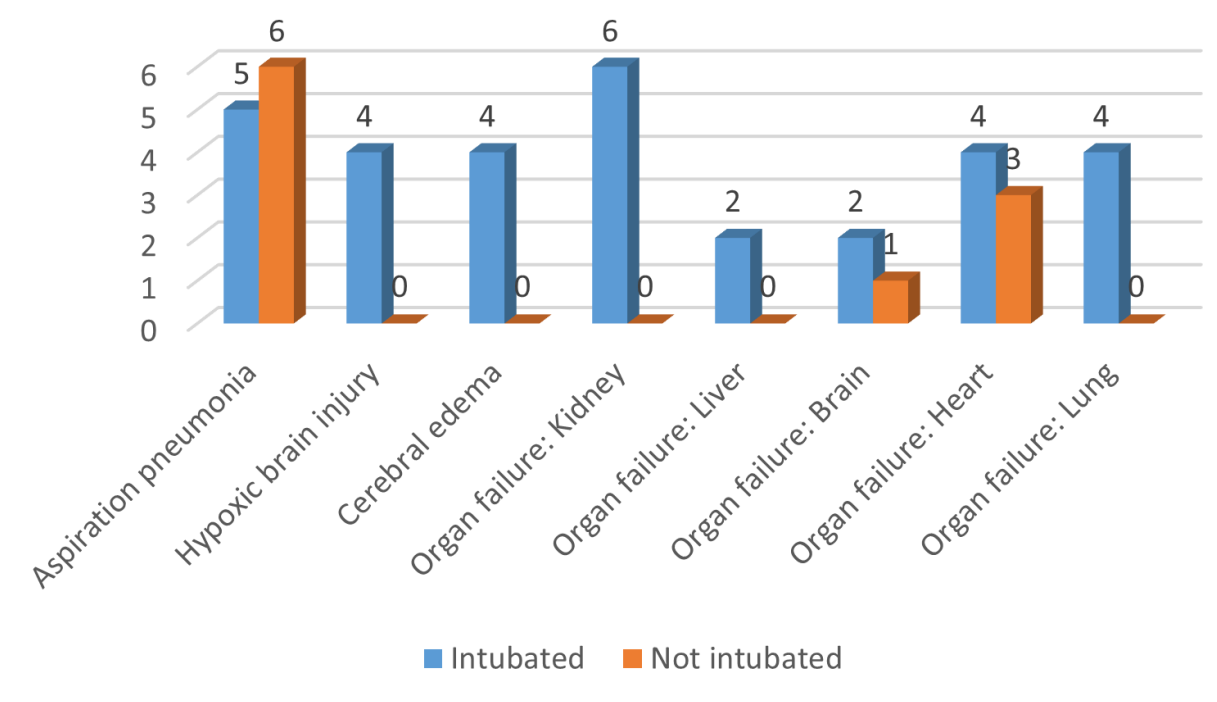

Fig. 3. Morbidity on admission 